\title{
Two Step Runge-Kutta-Nyström Methods for Oscillatory Problems Based on Mixed Polynomials
}

\author{
Beatrice Paternoster \\ Dipartimento di Matematica e Informatica \\ Universitá di Salerno, Italy \\ beapat@unisa.it
}

\begin{abstract}
We consider two step Runge-Kutta-Nyström methods for the numerical integration of $y^{\prime \prime}=f(x, y)$ having periodic or oscillatory solutions. We assume that the frequency $\omega$ can be estimated in advance. Using the linear stage representation, we describe how to derive two step Runge-Kutta-Nyström methods which integrate trigonometric and mixed polynomials exactly. The resulting methods depend on the parameter $\nu=\omega h$, where $h$ is the stepsize.
\end{abstract}

\section{Introduction}

We are concerned with the second order initial value problem

$$
y^{\prime \prime}(t)=f(t, y(t)), \quad y\left(t_{0}\right)=y_{0}, \quad y^{\prime}\left(t_{0}\right)=y_{0}^{\prime}, \quad y(t), f(t, y) \in R^{n},
$$

having periodic or oscillatory solutions, which describes many processes in technical sciences. Examples are given in celestial mechanics, molecular dynamics, seismology, and so on.

For ODEs of type (1), in which the first derivative does not appear explicitly, it is preferable to use a direct numerical method, instead of reducing the ODEs (1) into a first order system. An interesting and important class of initial value problems (1) which can arise in practice consists of problems whose solutions are known to be periodic, or to oscillate with a known frequency. Classical methods require a very small stepsize to track the oscillations and only methods which take advantage of some previous knowledge about the solution are able to integrate the system using a reasonable large stepsize. Therefore, efficiency can be improved by using numerical methods in which a priori information on the solution (as for instance, a good estimate of the period or of the dominant frequency) can be embedded.

In the following let us assume that a good estimate of the dominant frequency $\omega$ is known in advance. The aim is to exploit this extra information and to modify a given integration method in such a way that the method parameters are 'tuned' to the behavior of the solution. Such an approach has already been proposed by Gautschi in 1961 [6] for linear multistep methods for first-order differential equations in which the dominant frequencies $\omega_{j}$ are a priori known. 
Exploiting this idea, many numerical methods with coefficients depending on the predicted frequency are available in literature. Paternoster introduced RungeKutta-Nyström methods based on trigonometric polynomials [10], and methods resulting exact in phase when the high frequency component is produced by a linear part [11]. Coleman et al. considered methods based on mixed collocation 4. In the class of exponential-fitted methods many methods are available in literature. Ixaru in [7] focused on the numerical formulae associated with operations on oscillatory functions. Then many papers followed, we only cite here some of them 8141516]; see also the references therin.

In this paper we consider two step Runge-Kutta-Nyström methods for (1) having periodic or oscillatory solutions, for which a good estimate of the frequency is known in advance. We treat the TSRKN method as a composite linear multistep scheme, as done in Albrecht's approach [12]. Following the approach of 61012 , we define the trigonometric order of the methods. In section 2 we recall the conditions to obtain two step Runge-Kutta-Nyström methods which integrate algebraic polynomials exactly. In section 3 we give the definition of trigonometric order of the TSRKN method, and state the conditions to satisfy for integrating trigonometric polynomials exactly.

\section{Two Step Runge-Kutta-Nyström Methods Based on Algebraic Polynomials}

We consider the two step Runge-Kutta-Nyström methods (TSRK)

$$
\begin{aligned}
Y_{i-1}^{j}= & y_{i-1}+h c_{j} y_{i-1}^{\prime}+h^{2} \sum_{s=1}^{m} a_{j s} f\left(x_{i-1}+c_{s} h, Y_{i-1}^{s}\right), \quad j=1, \ldots, m \\
Y_{i}^{j}= & y_{i}+h c_{j} y_{i}^{\prime}+h^{2} \sum_{s=1}^{m} a_{j s} f\left(x_{i}+c_{s} h, Y_{i}^{s}\right), \quad j=1, \ldots, m, \\
y_{i+1}= & (1-\theta) y_{i}+\theta y_{i-1}+h \sum_{j=1}^{m} v_{j}^{\prime} y_{i-1}^{\prime}+h \sum_{j=1}^{m} w_{j}^{\prime} y_{i}^{\prime}+ \\
& h^{2} \sum_{j=1}^{m}\left(v_{j} f\left(x_{i-1}+c_{j} h, Y_{i-1}^{j}\right)+w_{j} f\left(x_{i}+c_{j} h, Y_{i}^{j}\right)\right), \\
y_{i+1}^{\prime}= & (1-\theta) y_{i}^{\prime}+\theta y_{i-1}^{\prime}+h \sum_{j=1}^{m}\left(v_{j}^{\prime} f\left(x_{i-1}+c_{j} h, Y_{i-1}^{j}\right)+w_{j}^{\prime} f\left(x_{i}+c_{j} h, Y_{i}^{j}\right)\right) .
\end{aligned}
$$

for the initial value problem (1). $\theta, v_{j}, w_{j}, v_{j}^{\prime}, w_{j}^{\prime}, a_{j s}, j, s,=1, \ldots, m$ are the coefficients of the methods, which can be represented by the Butcher array

$$
\begin{array}{c|cc|cccc} 
& & c_{1} & a_{11} & a_{12} & \cdots & a_{1 m} \\
\mathbf{c} & \mathbf{A} & c_{2} & a_{21} & a_{22} & \cdots & a_{2 m} \\
& \mathbf{v} & \vdots & \vdots & \vdots & & \vdots \\
\mathbf{w} & = & c_{m} & a_{m 1} & a_{m 2} & \cdots & a_{m m} \\
\hline \theta & v_{1} & v_{2} & \cdots & v_{m} \\
& & w_{1} & w_{2} & \cdots & w_{m} \\
\mathbf{v}^{\prime} & & v_{1}^{\prime} & v_{2}^{\prime} & \cdots & v_{m}^{\prime} \\
\mathbf{w}^{\prime} & & w_{1}^{\prime} & w_{2}^{\prime} & \cdots & w_{m}^{\prime}
\end{array}
$$


The TSRKN method (2), introduced in 13, was derived as an indirect method from the two step Runge-Kutta method presented in 9]. In comparison with classical one step Runge-Kutta-Nyström methods, TSRKN methods need a lower number of stages to rise to a given order of convergence. Indeed, advancing from $x_{i}$ to $x_{i+1}$, we only have to compute $Y_{i}$, because $Y_{i-1}$ have already been evaluated in the previous step. Therefore the computational cost of the method depends on the matrix $A$, while the vector $v$ adds extra degrees of freedom.

It is known that the method (2) is zero-stable if [13.

$$
-1<\theta \leq 1
$$

We treat formulas (2) by extending Albrecht's technique 12 to the numerical method we considered, as in [10,12]. According to this approach, we regard the TSRKN method (2) as a composite linear multistep scheme on a not equidistant grid.

$Y_{i-1}^{j}$ and $Y_{i}^{j}$ in (2) are called internal stages; $y_{i+1}$ and $y_{i+1}^{\prime}$ are the final stages, which give the approximation of the solution and its derivative of the solution in the step point $x_{i}$.

We associate a linear difference operator with each internal stage $Y_{i}^{J}$ of (2), in the following way:

$$
\mathcal{L}_{j}[z(x) ; h]=z\left(x+c_{j} h\right)-z(x)-h c_{j} z^{\prime}(x)-h^{2} \sum_{s=1}^{m}\left(a_{j s} z^{\prime \prime}\left(x+c_{s} h\right),\right.
$$

for $j=1, \ldots, m$, While the operator

$$
\begin{array}{r}
\overline{\mathcal{L}}[z(x) ; h]=z(x+h)-(1-\theta) z(x)-\theta z(x-h)-h\left(\sum_{j=1}^{m} v_{j}^{\prime} z^{\prime}(x-h)+\right. \\
\left.\sum_{j=1}^{m} w_{j}^{\prime} z^{\prime}(x)\right)-h^{2} \sum_{j=1}^{m}\left(v_{j} z^{\prime \prime}\left(x+\left(c_{j}-1\right) h\right)+w_{j} z^{\prime \prime}\left(x+c_{j} h\right)\right),
\end{array}
$$

is associated with the stage $y_{i+1}$ in (2). Finally

$$
\begin{aligned}
\overline{\mathcal{L}}^{\prime}[z(x) ; h]= & h z^{\prime}(x+h)-h(1-\theta) z^{\prime}(x)-\theta h z^{\prime}(x-h)- \\
& h^{2} \sum_{j=1}^{m}\left(v_{j}^{\prime} z^{\prime \prime}\left(x+\left(c_{j}-1\right) h\right)+w_{j}^{\prime} z^{\prime \prime}\left(x+c_{j} h\right)\right)
\end{aligned}
$$

is associated with the final stage $y_{i+1}^{\prime}$ in (2). It follows that

$$
\mathcal{L}_{j}[1 ; h]=\mathcal{L}_{j}[x ; h]=0, \quad j=1, \ldots, m,
$$

which implies that $y\left(x_{i}+c_{j} h\right)-Y_{i}^{j}=O(h)$ for $h \rightarrow 0$. Moreover

$$
\overline{\mathcal{L}}[1 ; h]=\overline{\mathcal{L}}^{\prime}[1 ; h]=\overline{\mathcal{L}}^{\prime}[x ; h]=0, \quad j=1, \ldots, m,
$$

If we annihilate $(6)$ on the function $z(x)=x$, then from $\overline{\mathcal{L}}[x ; h]=0$, it follows that

$$
\sum_{j=1}^{m}\left(v_{j}^{\prime}+w_{j}^{\prime}\right)=1+\theta
$$


which represents the consistency condition already derived in [9]13 which, together with (4), ensures that the TSRKN is convergent with order at least one. If (5) is identically equal to zero when $z(x)=x^{p}$, i.e. if $\mathcal{L}_{j}\left[x^{p} ; h\right]=0$, then

$$
\sum_{s=1}^{m} a_{j s} c_{s}^{p-2}=\frac{c_{j}^{p}}{p(p-1)}, \quad j=1, \ldots, m .
$$

Moreover, if (6) is equal to zero when $z(x)=x^{p}$, i.e. $\overline{\mathcal{L}}\left[x^{p} ; h\right]=0$, then

$$
\sum_{j=1}^{m}\left(v_{j}\left(c_{j}-1\right)^{p-2}+w_{j} c_{j}^{p-2}\right)=\frac{1-(-1)^{p} \theta}{p(p-1)}-\frac{(-1)^{p-1}}{p-1} \sum_{j=1}^{m} v_{j}^{\prime} .
$$

Finally, if we annihilate (7) on the function $z(x)=x^{p}$, then from $\overline{\mathcal{L}}^{\prime}\left[x^{p} ; h\right]=0$, it follows that

$$
\sum_{j=1}^{m}\left(v_{j}^{\prime}\left(c_{j}-1\right)^{p-2}+w_{j}^{\prime} c_{j}^{p-2}\right)=\frac{1-(-1)^{p-1} \theta}{(p-1)} .
$$

We can now give the following definitions:

Definition 1. An $m$-stage TSRKN method is said to satisfy the simplifying conditions $C_{2}(p)$ if its parameters satisfy

$$
\sum_{s=1}^{m} a_{j s} c_{s}^{k-2}=\frac{c_{j}^{k}}{k(k-1)}, \quad j=1, \ldots, m, k=1, \ldots, p .
$$

Definition 2. An m-stage TSRKN method (2) is said to satisfy the simplifying conditions $B_{2}(p)$ if its parameters satisfy

$$
\begin{gathered}
\sum_{j=1}^{m}\left(v_{j}\left(c_{j}-1\right)^{k-2}+w_{j} c_{j}^{k-2}\right)=\frac{1-(-1)^{k} \theta}{k(k-1)}-\frac{(-1)^{k-1}}{k-1} \sum_{j=1}^{m} v_{j}^{\prime}, \\
j=1, \ldots, m, k=1, \ldots, p .
\end{gathered}
$$

Definition 3. An $m$-stage TSRKN method is said to satisfy the simplifying conditions $B_{2}^{\prime}(p)$ if its parameters satisfy

$$
\sum_{j=1}^{m}\left(v_{j}^{\prime}\left(c_{j}-1\right)^{k-2}+w_{j}^{\prime} c_{j}^{k-2}\right)=\frac{1-(-1)^{k-1} \theta}{(k-1)}, \quad k=1, \ldots, p .
$$

$C_{2}(p), B_{2}(p)$ and $B_{2}^{\prime}(p)$ allow the reduction of order conditions of trees in the theory of two step RKN methods, which is under development by the author of this paper; moreover they also mean that all the quadrature formulas represented by the TSRKN method have order at least $p$, similarly as it happens in the theory of Runge-Kutta methods [3]. [112]:

The following theorem can be obviously proved by using Albrecht's theory 
Theorem 1. If $C_{2}(p), B_{2}(p)$ and $B_{2}^{\prime}(p)$ hold, then the m-stage TSRKN method (1.2) has order of convergence $p$.

Proof. $C_{2}(p), B_{2}(p)$ and $B_{2}^{\prime}(p)$ imply that all the stages of the method have order $p$ or, in Albrecht's terminology, that each stage in (2) has order of consistency $p$, so that the method has order of consistency $p$. In this case the method converges with order at least $p$.

It is worth mentioning that the conditions $C_{2}(p), B_{2}(p)$ and $B_{2}^{\prime}(p)$ are only sufficient conditions for the TSRKN method to have order $p$, but not necessary. Indeed the final stage must have order of consistency $p$, which is the condition $B_{2}^{\prime}(p)$, but it is not necessary that also the internal stages have order of consistency $p$. If all the stages have order of consistency $p$, then they are exact on any linear combination of the power set $\left\{1, x, x^{2}, \ldots, x^{p}\right\}$, and this implies that the TSRKN method results exact when the solutions of the system of ODEs(1) are algebraic polynomials. Moreover the simplifying conditions $C_{2}(p), B_{2}(p)$ and $B_{2}^{\prime}(p)$ are a constructive help for the derivation of new numerical methods within the class of TSRKN methods having a high order stage, and will be a useful basis for characterizing collocation TSRKN methods.

\section{Two Step Runge-Kutta-Nyström Methods Based on Trigonometric Polynomials}

Now, we can consider TSRKN methods which integrate ODEs (1) having periodic or oscillatory solutions, which can be expressed through trigonometric polynomials.

Let us suppose that a good approximation of the dominant frequency $\omega$ is known in advance, and that (4) and (8) hold, so that the method is convergent with order at least one.

Following Gautschi [6] and [1012, we state now the definition of trigonometric order.

Definition 4. The two step RKN method

\begin{tabular}{r|r}
$\mathbf{c}(\nu)$ & $\mathbf{A}(\nu)$ \\
& $\mathbf{v}(\nu)$ \\
$\theta$ & $\mathbf{w}(\nu)$ \\
& $\mathbf{v}^{\prime}(\nu)$ \\
& $\mathbf{w}^{\prime}(\nu)$
\end{tabular}

is said to be of trigonometric order q, relative to the frequency $\omega$, if the associated linear difference operators (5)-(7) satisfy 


$$
\begin{array}{lcc}
\mathcal{L}_{j}[1 ; h] & =\overline{\mathcal{L}}[1 ; h]=\overline{\mathcal{L}}^{\prime}[1 ; h]=0, & j=1, \ldots, m ; \\
\mathcal{L}_{j}[\cos r \omega x ; h]=\mathcal{L}_{j}[\sin r \omega x ; h]=0, & i=1, \ldots, m, \quad r=1, \ldots, q ; \\
\overline{\mathcal{L}}[\cos r \omega x ; h]=\overline{\mathcal{L}}[\sin r \omega x ; h]=0, & r=1, \ldots, q ; \\
\overline{\mathcal{L}}^{\prime}[\cos r \omega x ; h]=\overline{\mathcal{L}}^{\prime}[\sin r \omega x ; h]=0, & r=1, \ldots, q,
\end{array}
$$

with $\nu=\omega h$.

It is already known that methods with trigonometric order $q$ have algebraic order $2 q$ (see [610] for the definition of algebraic order) and therefore have order of convergence $2 q$.

It is easy to verify that a TSRKN method has trigonometric order $q$, according to Definition 4, if its parameters satisfy the following systems:

$$
\begin{aligned}
& A(q)=\left\{\begin{array}{l}
\sum_{s=1}^{m} a_{j s} \cos \left(r c_{s} \nu\right)=\frac{1-\cos \left(r c_{j} \nu\right)}{r^{2} \nu^{2}} \\
\sum_{s=1}^{m} a_{j s} \sin \left(r c_{s} \nu\right)=\frac{c_{j}}{r \nu}-\frac{\sin \left(r c_{j} \nu\right)}{r^{2} \nu^{2}}
\end{array} j=1, \ldots, s\right.
\end{aligned}
$$

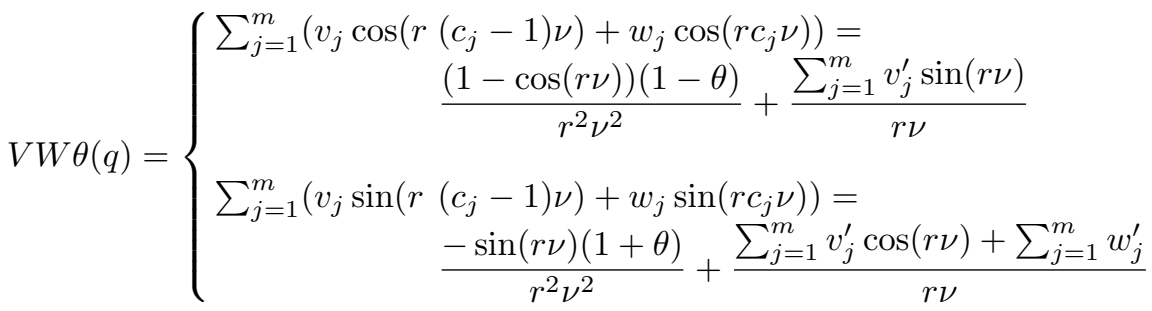

$$
\begin{aligned}
& V^{\prime} W^{\prime} \theta(q)=\left\{\begin{array}{l}
\sum_{j=1}^{m}\left(v_{j}^{\prime} \cos \left(r\left(c_{j}-1\right) \nu\right)+w_{j}^{\prime} \cos \left(r c_{j} \nu\right)=\frac{\sin (r \nu)(1+\theta)}{r \nu}\right. \\
\sum_{j=1}^{m}\left(v_{j}^{\prime} \sin \left(r\left(c_{j}-1\right) \nu\right)+w_{j}^{\prime} \sin \left(r c_{j} \nu\right)=\frac{(1-\theta)(1-\cos (r \nu)}{r \nu}\right.
\end{array}\right.
\end{aligned}
$$

for $r=1, \ldots, q$.

The following theorem states some constructive conditions to derive TSRKN methods giving exact solution (within the roundoff) error when the solution of ODEs (1) is a mixed polynomial which oscillates with frequency $\omega$.

Theorem 2. If the coefficients of the TSRKN method satisfy the conditions $C_{2}(p), B_{2}(p), B_{2}^{\prime}(p)$ and $A(q), V W \theta(q), V^{\prime} W^{\prime} \theta(q)$, then the TSRKN method integrates any linear combination of $\left\{1, x, x^{2}, \ldots, x^{p}, \cos \omega x, \sin \omega x, \cos 2 \omega x, \sin 2 \omega x\right.$, $\ldots, \cos q \omega x, \sin q \omega x\}$ exactly.

The construction of the methods requires the solutions of the linear systems $C_{2}(p), B_{2}(p), B_{2}^{\prime}(p)$ and $A(q), V W \theta(q), V^{\prime} W^{\prime} \theta(q)$, which is underdetermined. It 
is possible to solve the uncoupled linear systems after fixing some free parameters. Through symbolic computation it is possible to determine the analytical expressions of the remaining parameters of the method; for a high number of stages the involved systems have to be solved numerically.

\section{Conclusions}

Numerical methods for (1) having frequency-dependent parameters are quite widely used methods 45681011/141516], when a good approximation of the frequency $\omega$ to be fitted is a priori available. In this paper we design the approach to be used in the derivation of two step Runge-Kutta-Nyström methods in the case that only one frequency is fitted, but the development of TSRKN methods in which more frequencies are fitted can be considered as well. The linear stability analysis of these methods has not be considered in this paper, and has to follow the lines drawn in 58,4 .

Recently some authors [16] addressed the problem of how choosing the optimal value of the frequency to predict, and this new perspective enlarges the sphere of application of methods with $\nu$-dependent parameters, where $\nu$ is given by the product of the fitted frequency and the stepsize.

The coefficients of methods which are frequency-dependent involve combinations of trigonometric functions.

It is known that a common feature of this type of methods is that heavy cancelations occur during the evaluation of the coefficients from their closed form, which becomes increasingly severe as $\nu$ tends to 0 . Therefore, in the application, the expansions of the coefficients in powers of $\nu$, generated for example by Maple or Mathematica, is strongly recommended.

\section{References}

1. Albrecht, P.: Elements of a general theory of composite integration methods, Appl. Math. Comp. 31 (1989) 1-17.

2. Albrecht, P.: A new theoretical approach to RK methods, SIAM J. Numer. Anal. 24(2) (1987) 391-406.

3. Butcher, J.C.: The Numerical Analysis of Ordinary Differential Equations: RungeKutta and General Linear Methods, Wiley, New York (1987).

4. Coleman, J.P., Duxbury, S.C.: Mixed collocation methods for $y^{\prime \prime}=f(x, y)$, J. Comput. Appl. Math. 126 (2000) 47-75.

5. Coleman J.P., Ixaru, L.Gr.: P-stability and exponential-fitting methods for $y^{\prime \prime}=$ $f(x, y)$, IMA J. Numer. Anal. 16 (1996) 179-199.

6. Gautschi, W.: Numerical integration of ordinary differential equations based on trigonometric polynomials, Numer. Math. 3 (1961) 381-397.

7. Ixaru, L. Gr.: Operations on oscillatory functions, Comput. Phys. Comm. 105 (1997) 1-19.

8. Ixaru, L.Gr., Paternoster, B.: A conditionally P-stable fourth-order exponentialfitting method for $y^{\prime \prime}=f(x, y)$, J. Comput. Appl. Math. 106 (1999) 87-98.

9. Jackiewicz, Z., Renaut, R., Feldstein, A.: Two-step Runge-Kutta methods, SIAM J. Numer. Anal. 28(4) (1991) 1165-1182. 
10. Paternoster, B.: Runge-Kutta(-Nyström) methods for ODEs with periodic solutions based on trigonometric polynomials, Appl. Numer. Math. 28(2-4) (1998) 401-412.

11. Paternoster, B.: A phase-fitted collocation-based Runge-Kutta-Nyström method, Appl. Numer. Math. 35(4) (2000) 239-355.

12. Paternoster, B.: General two-step Runge-Kutta methods based on algebraic and trigonometric polynomials, Int. J. Appl. Math. 6(4) (2001) 347-362.

13. Paternoster, B.: Two step Runge-Kutta-Nyström methods for $y^{\prime \prime}=f(x, y)$ and Pstability, Computational Science - ICCS 2002, Lecture Notes in Computer Science 2331, Part III, P.M.A.Sloot, C.J.K.Tan, J.J.Dongarra, A.G.Hoekstra Eds., 459466, Springer Verlag, Amsterdam (2002).

14. Simos, T.E.: An exponentially-fitted Runge-Kutta method for the numerical integration of initial-value problems with periodic or oscillating solutions, Comput. Phys. Comm. 115 (1998) 1-8.

15. Vanden Berghe, G., De Meyer, H., Van Daele, M., Van Hecke, T., Exponentiallyfitted Runge-Kutta methods, J. Comput. Appl. Math. 125 (2000) 107-115.

16. Vanden Berghe, G., Ixaru, L. Gr., De Meyer, H.: Frequency determination and step-length control for exponentially-fitted Runge-Kutta methods, J. Comput. Appl. Math. 132 (2001) 95-105. 